\title{
An example of resonances, coherent structures and topological phase transitions - the origin of the low frequency broadband spectrum in the auroral zone
}

\author{
T. Chang \\ Center for Space Research, Massachusetts Institute of Technology Cambridge, MA 02139, USA
}

Received: 29 June 2000 - Accepted: 29 August 2000

\begin{abstract}
We consider the phenomena of intermittent turbulence in magnetized space plasmas from the point of view of topological phase transitions involving the merging and interactions of anisotropic coherent structures. The stochastic behavior of these coherent plasma structures can undergo complex changes as the dynamic system evolves, similar to those commonly observed in (first and second order) equilibrium phase transitions. When conditions are favorable, such topological entities can evolve into a state of forced and/or self-organized criticality (FSOC). As an example, we apply these ideas to the understanding of the origin of the commonly observed broadband power-law low frequency electric field spectral densities and the characteristic filamentary current structures in the auroral zone. The broadband turbulence can provide efficient resonant energization of the ionospheric oxygen ions.
\end{abstract}

\section{Introduction}

Most of the observed turbulent space plasma processes are intrinsically intermittent with at least two distinctly different time scales, one time scale associated with the intermittency, which is very short, and an evolutionary time scale, which is much longer in duration. This has led Chang (1992) to suggest that the behavior for intermittent turbulent magnetized plasma processes is related to the phenomenon of forced and/or self-organized criticality (FSOC), based on the concepts of path integrals and the dynamical renormalization-group. Subsequently, Chang $(1998,1999)$ constructed physically meaningful topological models to describe the basic interactions for such processes in the context of the formation and evolution of the coherent structures generated near plasma resonances, and the fast interactions and relaxation diffusion among these structures. From first principles, analogies were established between the concepts involving topological phase transitions for dynamical systems far from

Correspondence to: T. Chang (tsc@ space.mit.edu) equilibrium and those in equilibrium phase transitions. As an example, we apply these ideas and techniques to arrive at a basic understanding of the origin of the commonly observed broadband low frequency broken-power-law spectra involving the intermittent turbulent electric field fluctuations in the auroral zone.

\section{The concepts of coherent structures in space plasmas}

Most field theoretical discussions begin with the concept of the propagation of waves. For example, in the MHD formulation, one can combine the basic equations and obtain the following convective expressions:

$\rho \frac{d \boldsymbol{V}}{d t}=(\boldsymbol{B} \cdot \nabla) \boldsymbol{B}+\ldots$
$\frac{d \boldsymbol{B}}{d t}=(\boldsymbol{B} \cdot \nabla) \boldsymbol{V}+\ldots$

where the ellipsis represent the effects of the anisotropic pressure tensor, the compressible and dissipative effects, and all notations are standard. Equations $(1,2)$ allow the propagation of Alfvén waves. For such waves to propagate, the propagation vector $\boldsymbol{k}$ must contain a field-aligned component, i.e. $\boldsymbol{B} \cdot \boldsymbol{\nabla} \rightarrow i \boldsymbol{k} \cdot \boldsymbol{B} \neq 0$. However, at sites where the parallel component of the propagation vector vanishes (i.e. at the resonance sites), the fluctuations are localized. Around these resonance sites (usually in the form of curves in physical space), it may be shown that the fluctuations are held back by the background magnetic field, forming anisotropic coherent structures in the form of flux tubes (Chang, 1998, 1999) (see Fig. 1).

As the coherent structures migrate toward each other, there will be interactions. Sometimes these interactions can lead to the merging of the structures. Moreover, the interactions will produce new plasma fluctuations and new plasma resonances which are the seeds of new coherent structures. Consequently, an intricate process of merging, interaction and evolution of these topological structures results. 


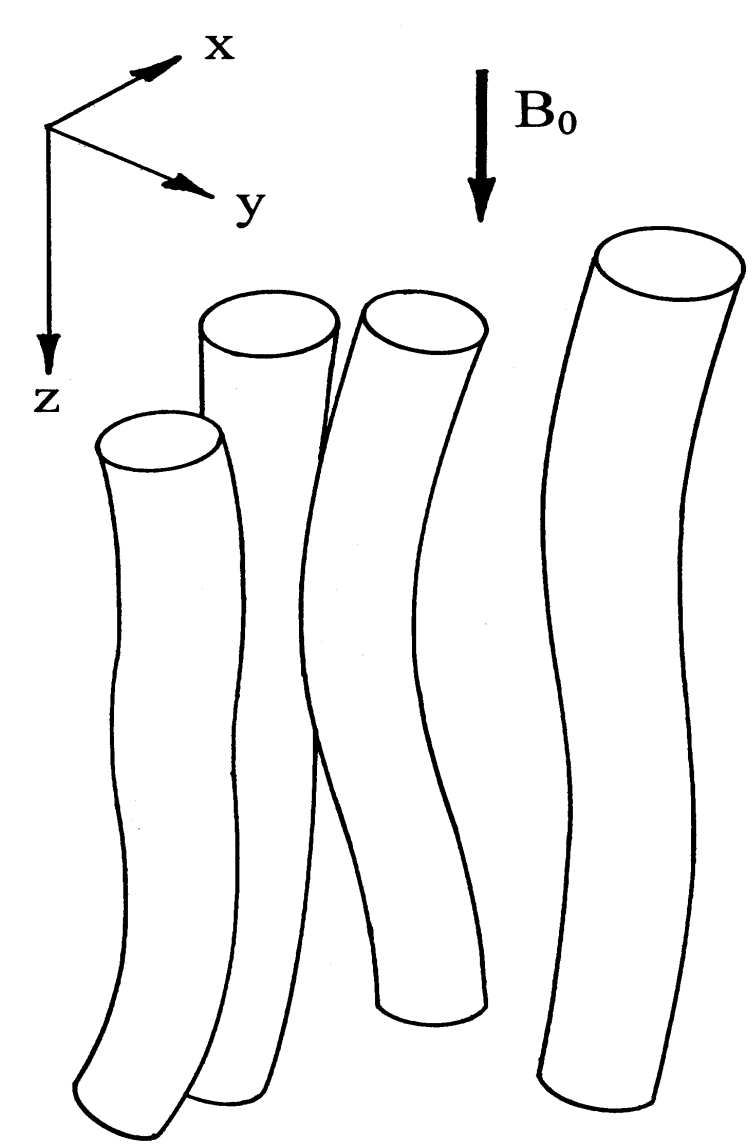

Fig. 1. Wiggling and meandering field-aligned current filaments.

In a turbulent MHD plasma, it has been suggested by Taylor (1974) that in a relaxed state, such a structure would be approximately force-free (i.e. $\boldsymbol{j} \times \boldsymbol{B}=0$ ) due to the approximate conservation of the coarse-grained helicity, defined as $K=\int \boldsymbol{A} \cdot \boldsymbol{B} d V$ integrated over the coherent structure, where $\boldsymbol{j}$ and $\boldsymbol{B}$ are the current density vector and magnetic field, and $\boldsymbol{A}$ is the vector potential. (We note that if a coherent structure is not simply connected and/or that the $K$ integral is not confined by a closed flux surface, the above discussions on helicity will need to be more carefully stated to ensure gauge invariance.)

We now consider the example of low frequency auroral turbulence. For the auroral geometry and low beta plasma, the force free condition is approximately satisfied by the scalar condition, $\boldsymbol{B} \cdot \nabla j=0$, where $\boldsymbol{B}$ is approximately in the direction of the geomagnetic field and $j$ is approximately the intensity of the field-aligned current. It can be shown that, with the inclusion of the kinetic effects through the anisotropic pressure terms and the generalized Ohm's law, the above results are still approximately valid. We have, then, approximately,

$B_{0} \frac{\partial j_{z}}{\partial z}=-\left(\delta B_{x} \frac{\partial}{\partial x}+\delta B_{y} \frac{\partial}{\partial y}\right) j_{z}+\ldots$ where $B_{0}$ is the geomagnetic field, $z$ is the field-aligned direction, $(x, y)$ are orthogonal coordinates normal to $z, j_{z}$ is the field-aligned current density, and the ellipsis represent the other modifying effects. Writing $(-\partial \psi / \partial x, \partial \psi / \partial y)=$ $\left(\delta B_{y}, \delta B_{x}\right)$ for the fluctuating components of the magnetic field components in the $(x, y)$ directions to satisfy $\nabla \cdot \boldsymbol{B}=0$, $j_{z}$ and $\psi$ are determined by Eq. (3) and the Ampere's law. A simple example would be the class of circularly cylindrical solutions of $\psi(r)$ and $j_{z}(r)$. Generally, the solutions would be much more complicated due to of the variabilities of the local conditions of the plasma and the three-dimensional geometry. Thus, we envision the existence of bundles of sporadically generated and nearly field-aligned localized current filaments (Fig. 1). These filaments (carrying upward or downward currents) wiggle and meander within the auroral zone. In the acceleration region, the total current carried by the filaments would be upward; the reverse is true in the return current and/or black auroral region.

When filamentary current structures of the same polarity migrate toward each other, strong current sheets are generated. In the ionosphere, these could be the origins of the observed small auroral arcs. When they move very close to each other, the current sheets can become very intense (Fig. 2). (An example of a two-dimensional analog has been simulated by $\mathrm{Wu}$ and Chang, 2000). At this point, a number of possible follow-up scenarios may result. Firstly, the thinness of the intense current sheets enhances the electron inertia effect in the generalized Ohm's law. This would provide the possibility of collisionless tearing instabilities (Seyler, 1990). On the other hand, if the sizes of the current filaments are of the order of the electron skin depth, then the thickness of the intense current sheets of the interaction regions among the current filaments can be even smaller and may approach that of the order of the local ion gyroradius. The ions would then become effectively unmagnetized and the electrons remain magnetized; thus, enhancing the electron Hall currents. As the electrons travel across the magnetic field lines, they would excite whistler fluctuations. In analogy to the Alfvén resonances, singularities of $k_{\|}=\boldsymbol{k} \cdot \boldsymbol{B}=0$ generally can develop, at which whistler fluctuations cannot propagate. These "whistler resonances" can provide the nuclear sites for the emergence of coherent whistler structures, which is the analog of the coherent Alfvénic structures, but with much smaller scales. The intermittent turbulence resulting from the intermixing and interactions of the coherent whistler structures in the intense current sheet region can then provide the coarse-grain averaged dissipation that allows the filamentary current structures to merge, interact, or breakup. In addition to the above scenarios, other plasma instabilities may set in when conditions are favorable.

\section{Forced and/or self-organized criticality (FSOC)}

In proposing a new paradigm for the understanding of the dynamics of the magnetotail, Chang $(1998,1999)$ suggested that the coherent structures which arose from the Alfvén 


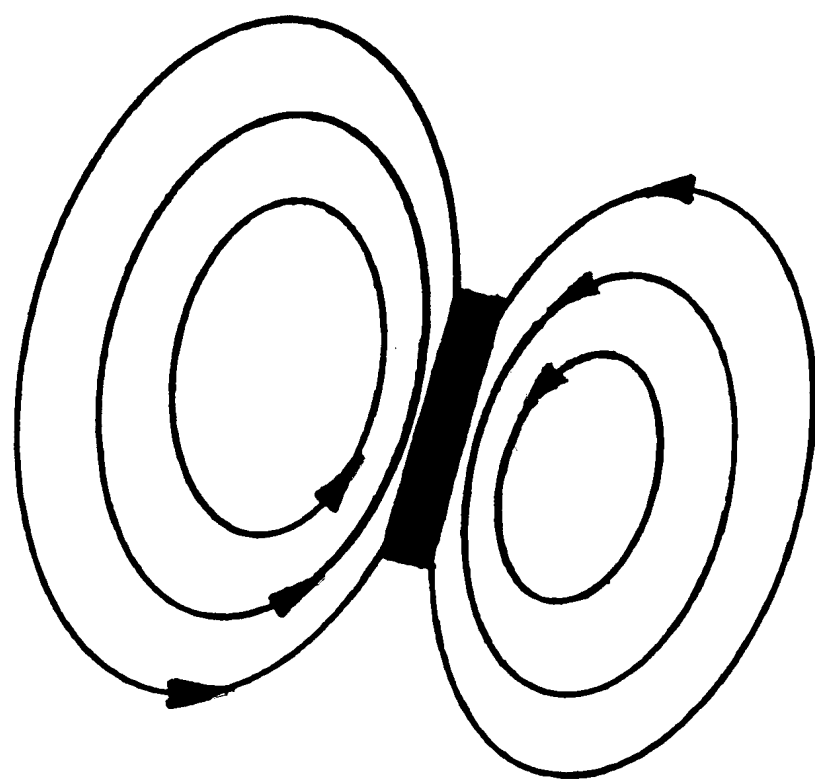

Fig. 2. Cross sectional view of two merging current filaments of the same polarity. Contours are $\psi=$ const and arrows indicate directions of perturbed magnetic field in the $(x, y)$-plane. Blackened area is an intense current.

resonances in the neutral sheet region of the magnetotail may evolve into a forced and/or self-organized critical state (FSOC). We suggest that a similar scenario may be applied to our example in the auroral zone. Here, the coherent filamentary current structures that emerge from the Alfvénic resonances can merge and/or become unstable (because of the whistler turbulence induced within the intense current sheets, finite electron inertia and other possibilities discussed above), intermix and produce new resonance sites and new filamentary structures. When they evolve into a forced and/or self-organized critical state, power-law frequency $(\omega)$ and mode number $(k)$ spectra will result (including those for the associated velocity and electric field fluctuations). The demonstration of such possibilities was based on the concepts of the modern theory of critical phenomena and has been studied by Chang $(1992,1998,1999)$ for the magnetotail scenario via the formalisms of path integrals and the dynamic renormalization-group (Chang et al., 1978; Chang et al., 1992) (see Sec. 4.). This would then provide a possible explanation for the observed ubiquitously broadband power-law spectral densities of the electric field fluctuations. The power-law index is expected to change across the various characteristic frequencies or length scales of the plasma medium (such as the ion gyrofrequencies or ion gyroradii; see, e.g. Fig. 8 of Chang, 1999). Although the origin of the field-aligned current structures is assumed to arise from the consideration of plasma resonances and magnetic fluctuations, the resulting plasma dynamics in the auroral zone will generally be encompassed in the electric field structures. Because of the coherent and non-propagating nature of the

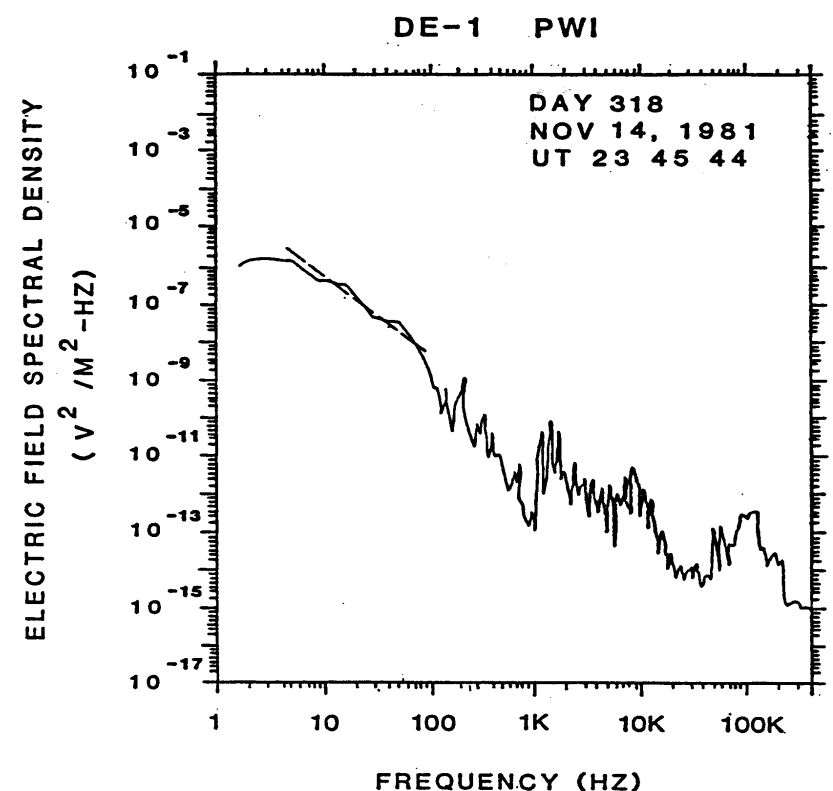

Fig. 3. Typical electric field spectral density in the diffuse auroral region at a geocentric altitude of $2 \mathrm{R}_{\mathrm{E}}$. The oxygen gyrofrequency is around $6 \mathrm{~Hz}$. Mellot and Gurnett (private communication).

current structures, the associated electric field generally can carry a significant electrostatic component. Moreover, the perpendicular components of the electric field fluctuations would generally dominate over the field-aligned component, even with the effect of the electron inertia.

At the same time, the whistler coherent structures, which may have provided the dissipation required for the merging and breakups of the filamentary current structures, may evolve into another FSOC state (however, generally with a different power-law index, for the electric field spectral density). They may contribute to a fraction of the observed VLF emissions with the characteristic lower hybrid cutoffs. Because of the power-law nature of the combined Alfvénic and whistler spectra (with negative indices) for forced and/or self-organized critical states, the intensities of the electric field spectral density may be expected to become smaller and smaller at higher and higher frequencies. At higher frequencies, such as the range of the lower hybrid frequency, other plasma instabilities may set in and provide spectral humps in the otherwise broadband spectrum (Fig. 3). Note, we are now discussing frequencies below, above and in the range of the ion gyrofrequencies.

It has long been recognized that low frequency electric field fluctuations are responsible for the energization of the ionospheric oxygen ions in the auroral zone (Chang et al., 1986). The broadband power-law characteristics of the electric field fluctuations discussed above can conveniently provide the continuous resonant energization of the ions as they evolve upward along the field lines. The perpendicular diffu- 
sion coefficient to the lowest order would then simply be

$D_{\perp}=\frac{q^{2}}{m^{2}} \int d \omega d^{3} k \xi(\boldsymbol{k}, t) F(\boldsymbol{k}, t, \boldsymbol{v})$,

where $\xi$ is the electric field spectral density and $F$ is the resonance function which accommodates the required Doppler shifts for resonance interaction and stochastic broadening of interactions among the ions and fluctuations.

\section{Symmetry breaking and topological phase transitions}

For nonlinear stochastic systems near criticality, the correlations among the fluctuations of the random dynamical fields are extremely long-ranged and there exist many correlation scales. The dynamics of such systems are notoriously difficult to handle, either analytically or numerically. On the other hand, since the correlations are extremely long-ranged, it is reasonable to expect that the system will exhibit some sort of invariance under scale transformations. A powerful technique that utilizes this invariance property is the technique of the dynamic renormalization-group (Chang et al., 1978, 1992, and references contained therein). As it is described in these references, based on the path integral formalism, the behavior of a nonlinear stochastic system far from equilibrium may be described in terms of a "stochastic Lagrangian $L$ ". Then, the renormalization-group (coarsegraining) transformation may be formally expressed as:

$\frac{\partial L}{\partial \ell}=R L$

where $R$ is the renormalization-group (coarse-graining) transformation operator and $\ell$ is the coarse-graining parameter for the continuous group of transformation. It will be convenient to consider the state of the stochastic Lagrangian in terms of its parameters $\left\{P_{n}\right\}$. Equation (5), then, specifies how the Lagrangian, $L$, flows (changes) with $\ell$ in the affine space spanned by $\left\{P_{n}\right\}$ (Fig. 4).

Generally, there exists a number of fixed points (singular points) in the flow field, where $\partial L / \partial \ell=0$. At a fixed point ( $L^{*}$ or $L^{* *}$ in Fig. 4), the correlation length should not be changing. However, the renormalization-group transformation requires that all length scales must change under the coarse-graining procedure. Therefore, to satisfy both requirements, the correlation length must be either infinite or zero. When it is at infinity, the system is, by definition, at criticality. The alternative trivial case of zero correlation length will not be considered here.

To study the stochastic behavior of a nonlinear dynamical system near a particular criticality (e.g. the one characterized by the fixed point $L^{*}$ ), we can linearize the renormalizationgroup operator $R$ about $L^{*}$. The mathematical consequence of this approximation is that, close to criticality, certain linear combinations of the parameters that characterize the stochastic Lagrangian $L$ will correlate with each other in the form of power laws. This includes, in particular, the $(k, \omega)$, i.e. mode number and frequency, spectra of the correlations of the various fluctuations of the dynamic field variables. In addition,

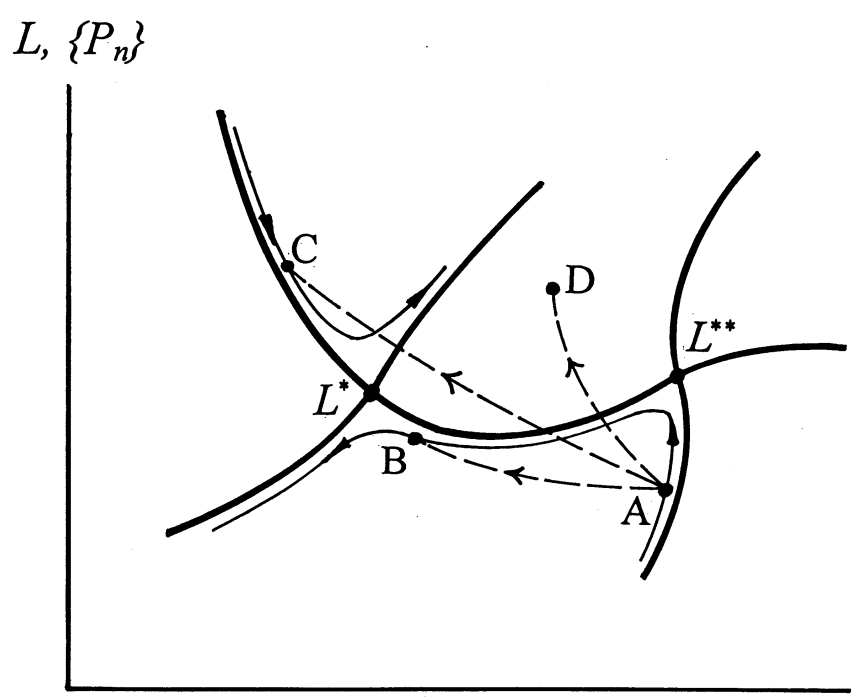

Fig. 4. Phase space (affine space) for the stochastic Lagrangian $L\left(\left\{P_{n}\right\}\right)$. Heavy solid lines are separactrices passing through the fixed points $L^{*}, L^{* *}$ under the renormalization-group (coarse graining) transformation. Light solid lines are renormalization-group tarjectories and thin solid arrows indicate the direction of increasing $\ell$. Broken lines and flaring arrows indicate paths and directions of the dynamic evolution of the stochastic system.

it can be demonstrated from such a linearized analysis that generally, only a small number of (relevant) parameters are needed to characterize the stochastic state of the system near criticality (i.e. low-dimensional behavior; see Chang, 1992).

As the dynamical system evolves in time, a number of transitional scenarios are possible. For example, the system may evolve from a critical state $A$ (characterized by $L^{* *}$ ) to another critical state $B$ (characterized by $L^{*}$ ), as shown in Fig. 4. In this case, the system may evolve continuously from one critical state to another. Such type of symmetry breaking can lead to the continuously varying scaling indices and multifractal characteristics.

On the other hand, the evolution from the critical state $A$ to critical state $C$, as shown in Fig. 4, would probably involve a dynamical instability characterized by a first- orderlike topological phase transition because the dynamical path of evolution of the stochastic system would have to cross over a couple of renormalization-group separatrices (shown as heavy solid lines). Alternatively, a dynamical system may evolve from a critical state $A$ to a state $D$ (as shown in Fig. 4), which may not be situated in a regime dominated by any of the fixed points; in such a case, the final state of the system will no longer exhibit any of the characteristic properties that are associated with criticality.

\section{Summary}

A theory of topological phase transitions is described to address the phenomena of intermittent turbulence in space plas- 
mas. The theory is based on the concepts of resonances and coherent structures. When conditions are favorable, the coherent structures may merge, interact and evolve into forced and/or self-organized critical states (FSOC). An example is given to provide a possible explanation of the development of broadband power-law electric field spectral densities in the auroral zone. Such low frequency intermittent turbulence in the auroral zone can efficiently accelerate the ionospheric oxygen ions (Chang et al., 1986; André et al., 1988.)

Acknowledgement. The author thanks M. André, G. Consolini, G. Ganguli, P. Kintner, C. Seyler, S. W. Y. Tam and C. C. Wu for useful discussions. Research is supported by grants from AFOSR, NSF and NASA.

\section{References}

André, M., Koskinen, H., Matson, L., and Erlandson, R., Local transverse ion energization in and near the polar cusp, Geophys. Res. Lett., 15, 107-110, 1988.

Chang, T., Low-dimensional behavior and symmetry breaking of stochastic systems near criticality - can these effects be observed in space and in the laboratory?, IEEE Trans. On Plasma Science, 20, 691-694, 1992.

Chang, T., Sporadic localized reconnections and multiscale intermittent turbulence in the magnetotail, in Geospace Mass and Energy Flow, Geophysical Monograph, No. 104, edited by J. L.
Horwitz, D. I. Gallagher, and W. K. Peterson, (American Geophysical Union, Washington, DC), pp. 193-199, 1998.

Chang, T., Self-organized criticality, multi-fractal spectra, sporadic localized reconnections and intermittent turbulence in the magnetotail, Phys. Plasmas, 6, 4137-4145, 1999.

Chang, T., Crew, G. B., Hershkowitz, N., Jasperse, J. R., Retterer, J. M., and Winningham, J. D., Transverse acceleration of oxygen ions by electromagnetic ion cyclotron resonance with broadband left-hand polarized waves, Geophys. Res. Lett., 13, 636639, 1986.

Chang, T., Nicoll, J. F., and Young, J. E., A closed-form differential renormalization-group generator for critical dynamics, Phys. Lett., 67A, 287-290, 1978.

Chang, T., Vvedensky, D. D., and Nicoll, J. F., Differential renormalization- group generators for static and dynamic critical phenomena, Phys. Reports, 217, 279-362, 1992.

Consolini, G. and Chang, T., Magnetic field topology and criticality in geotail dynamics: relevance to substorm phenomena, Space Science Rev., in press, 2000.

Seyler, C. E., A mathematical model of the structure and evolution of discrete auroral arcs, J. Geophys. Res., 95, 17199-17215, 1990.

Taylor, J., Relaxation of toroidal plasma and generation of reverse magnetic fields, Phys. Rev. Lett., 33, 1139-1141, 1974.

Wu, C. C. and Chang, T., 2D MHD simulation of the emergence and merging of coherent structures, Geophys. Res. Lett., 27, 863866, 2000. 